\title{
Relation of granulomas to lymphatic vessels in Crohn's disease
}

\author{
E E Mooney, J Walker, D O’B Hourihane
}

\begin{abstract}
Aims-To examine the relation between granulomas and lymphatic vessels in Crohn's disease.

Methods-Formalin fixed, paraffin wax embedded sections were selected from surgical resection specimens from 10 cases diagnosed as Crohn's disease. The block that showed the most granulomas was selected from each case. Sections $5 \mu \mathrm{m}$ thick were immunostained with antibodies directed against the endothelial markers factor VIII related antigen and Ulex europaeus lectin, and against the vascular wall components collagen IV and laminin. Granulomas were counted on each slide in the serosa, muscularis propria, submucosa, and mucosa. In each area granulomas were classified according to their relation to lymphatic or blood vessels. Results-Overall, an average of $46 \cdot 1 \%$ of granulomas (range 15.3-90.4\%) was related to lymphatic vessels, with the majority of these being adjacent to the vessel, rather than in the lumen or distorting the wall. A smaller percentage $(10 \cdot 1 \%$, range $2 \cdot 4-25 \cdot 8 \%$ ) was related to blood vessels. Conclusions-A significant proportion of granulomas in Crohn's disease is associated with lymphatic vessels. Blood vessel involvement may be a secondary phenomenon, rather than the primary event. (f Clin Pathol 1995;48:335-338)
\end{abstract}

Keywords: Granuloma, lymphatic vessels, Crohn's disease.

Crohn's disease is a multisystem disease characterised by non-caseating granulomas. Its aetiology remains obscure, although granulomatous vasculitis has been suggested as the significant pathogenic mechanism. ${ }^{1}$ Dilated lymphatic vessels are characteristic of Crohn's disease $^{2}$ and the localisation of lymphoid aggregates around them has been noted. A vasculitis - that is, an arteritis, is a feature of a minority of cases of Crohn's disease ${ }^{3}$ and the hypothesis that Crohn's disease is a primary vasculitis has not found general acceptance. ${ }^{4-6}$

In reporting cases of resection specimens of Crohn's disease submitted to our laboratory we noted that a large number of the characteristic loosely formed granulomas seemed to be intimately related to thin-walled dilated channels presumed to be lymphatic in nature. This study was designed to explore that relationship.

\section{Methods}

This study was based on a selection of 10 cases from surgical resection specimens diagnosed as
Crohn's disease. All tissues had been fixed in $10 \%$ buffered formalin and embedded in paraffin wax. Original diagnostic slides were reviewed and the diagnosis of Crohn's disease confirmed. In addition to granulomas, all cases showed transmural inflammation, submucosal oedema/fibrosis and mucosal ulceration. One block from each case which showed the greatest degree of granulomatous inflammation, and relative preservation of the mucosa, was selected for immunohistochemistry. Nine blocks were of ileum and one was of colon. Immunohistochemistry was performed on sections $5 \mu \mathrm{m}$ thick using a Streptavidin peroxidase technique as follows: antibody to factor VIII related antigen (polyclonal; Dako, Glostrup, Denmark) was used at a dilution of 1 in 400 after pretreatment with trypsin for 15 minutes; antibody to Ulex europaeus lectin (Vector Laboratories, Burlingame, California, USA) at a dilution of 1 in 200; antibody to collagen type IV (monoclonal; Dako) at a dilution of 1 in 50 after pretreatment with trypsin for 30 minutes; and antibody to laminin (monoclonal; Sigma, St Louis, Missouri, USA) at a dilution of 1 in 2000 after pretreatment with pepsin for 30 minutes. In addition, immunostaining for CD68 (monoclonal PG-M 1; Dako) was performed at a dilution of 1 in 80 after pretreatment with trypsin for 15 minutes.

For each region of the bowel wall (serosa, muscularis propria, submucosa, mucosa), the number of granulomas was counted. For the purpose of this study, a granuloma was defined as a localised aggregate of histiocytes with or without giant cells, or an aggregate of lymphocytes and histiocytes where the latter made up the majority of cells present. On staining with haematoxylin and eosin, vessels were judged to be either lymphatic or blood vessels principally according to the thickness of their wall related to luminal size, and to luminal contents, red cells indicating a thin-walled blood vessel. On immunohistochemistry, these morphological features were assessed in combination with the staining pattern in designating a vessel. Granulomas were assessed for location: where a granuloma was present in the lumen of a lymphatic vessel, with no obvious attachment to the wall, it was classified as "luminal". Where abutting on the wall, but not protruding into the lumen, it was classified as "adjacent", and distortion of the wall was a third diagnostic category. Granulomas classified as "related to blood vessels" were those involved in a granulomatous arteritis, or where there was an intimate relation between the granuloma and the adventitia and/or media of a blood vessel. Where there was no obvious relation to a blood or a lymphatic vessel, the designation "not otherwise specified" was applied. 


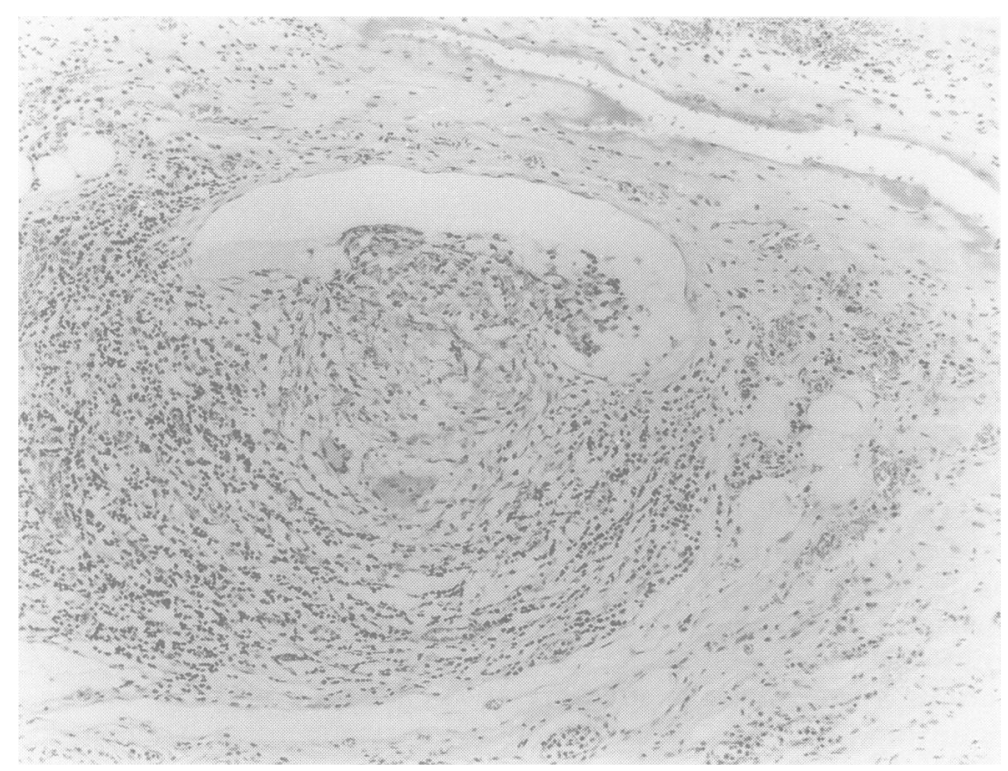

Figure 1 Loosely formed granuloma impinging on and distorting the wall of a lymphatic channel.

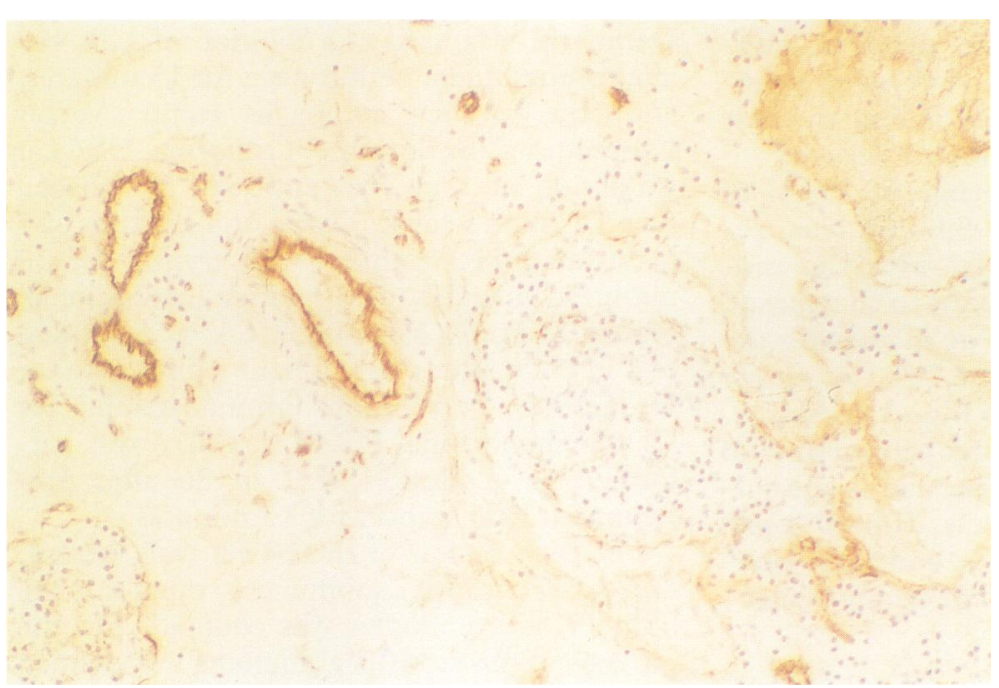

Figure 2 Positivity for factor VIII is strong in the three blood vessels (left) and weak and focal in the lymphatic channel (right), which contains a granuloma (immunostain for factor VIII).

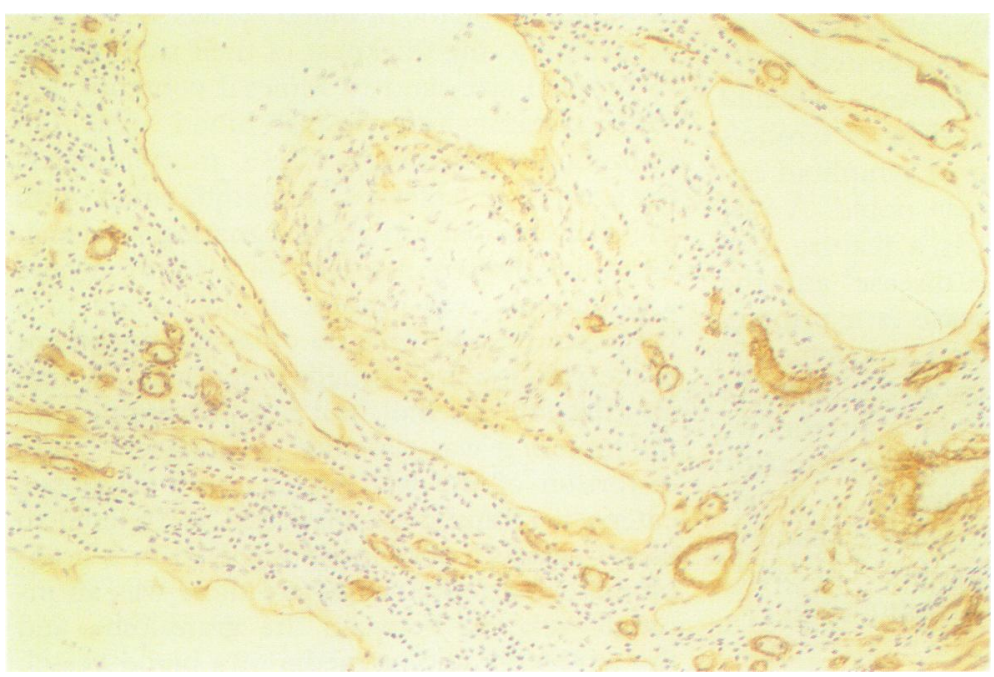

Figure 3 Staining for laminin is strongly positive in blood vessels, and shows minimal staining in lymphatic vessels, one of whose walls is disrupted by a granuloma (immunostain for laminin).
To establish that these aggregates of histiocytes were granulomas, 10 consecutive cases of ulcerative colitis resection specimens, comprising a total of 109 slides of colon, were reviewed. Clinical notes were examined and in all cases the clinical and (where radiology was performed) radiological diagnoses were consistent with the diagnosis made on macroscopic and microscopic examination of the specimen.

\section{Results}

Haematoxylin and eosin stained slides showed granulomas in the lumina of thin-walled channels, adjacent to these channels and disrupting their walls. The granulomas typically had a central aggregate of histiocytes, minimal stroma and a peripheral cuff of lymphocytes (fig 1).

Staining for factor VIII related antigen showed a thin, weak and focally discontinuous positivity in lymphatic vessels, in contrast to the thicker strong positivity seen in blood vessels (fig 2). Staining for Ulex europaeus lectin was similar in intensity in lymphatic and blood vessels, but was thinner in lymphatic vessels, and also showed focal discontinuity. Antibodies to collagen IV and laminin were diffusely positive around blood vessels, with occasional weak staining of the walls of larger lymphatic vessels, especially with laminin (fig 3). The histiocytic nature of the cells in the granulomas was confirmed by positive staining for CD68.

The number of granulomas identified in each case is shown in the table. This table shows the absolute numbers of granulomas seen on each slide. Category II (related to lymphatics) includes all granulomas that were classified as luminal, adjacent to lymphatics, or distorting the wall of a lymphatic vessel. Use of the endothelial antibodies resulted in confirmation of the original light microscopic impression, with almost all cases showing an increase in the relative number of granulomas that could be classified as associated with lymphatic vessels. Further assessment using the vascular wall markers enabled a smaller proportion to be classified as related to blood vessels. In some cases this was because of reclassification of a vessel thought to be lymphatic on the basis of endothelial marker staining, but use of collagen IV and laminin also enabled visualisation of small vessels disrupted by granulomatous inflammation and classified on staining with haematoxylin and eosin as "not otherwise specified".

The granulomas were for the most part small and loosely formed, and it was possible to follow the same granuloma through sequential levels in only a minority of cases. The absolute number of granulomas therefore differs on the different slides, but the general trend was maintained.

Of the granulomas that were felt to be related to lymphatic vessels, the majority (49\%) were categorised as "adjacent". The remainder were divided almost equally between the other two categories. In some cases the same granuloma could be seen in the lumen on one slide and the point of disruption of the wall became apparent on the following slides which acted as levels. Thus, the same granuloma may have been classified in different categories for the different stains. 
Absolute numbers of granulomas

\begin{tabular}{|c|c|c|c|c|c|c|c|c|c|c|c|c|c|c|c|}
\hline \multirow[b]{2}{*}{ Case No. } & \multicolumn{3}{|c|}{$H+E$} & \multicolumn{3}{|c|}{ Factor VIII } & \multicolumn{3}{|c|}{ Ulex } & \multicolumn{3}{|c|}{ Collagen IV } & \multicolumn{3}{|c|}{ Laminin } \\
\hline & $I$ & $I I$ & III & $I$ & II & $I I I$ & $I$ & $I I$ & $I I I$ & $I$ & $I I$ & $I I I$ & $I$ & $I I$ & $I I I$ \\
\hline 1 & 20 & 23 & 0 & 4 & 31 & 0 & 3 & 45 & 0 & 5 & 22 & 1 & 8 & 40 & 7 \\
\hline 2 & 8 & 4 & 4 & 3 & 6 & 2 & 6 & 4 & 2 & 4 & 7 & 1 & 9 & 11 & 5 \\
\hline 3 & 7 & 10 & 0 & 3 & 10 & 0 & 9 & 14 & 1 & 9 & 13 & 6 & 9 & 12 & 3 \\
\hline 4 & 42 & 49 & 5 & 21 & 39 & 5 & 35 & 31 & 9 & 27 & 42 & 6 & 10 & 48 & 5 \\
\hline 5 & 7 & 7 & 0 & 12 & 4 & 2 & 7 & 8 & 0 & 6 & 5 & 2 & 9 & 9 & 0 \\
\hline 6 & i & 5 & 0 & 1 & 8 & 0 & 1 & 4 & 0 & 0 & 11 & 1 & 0 & 10 & 0 \\
\hline 7 & 6 & 0 & 2 & 0 & 3 & 1 & 3 & 2 & 1 & 2 & 2 & 2 & 2 & 4 & 3 \\
\hline 8 & 16 & 1 & 3 & 9 & 5 & 1 & 13 & 6 & 3 & 9 & 7 & 2 & 8 & 3 & 4 \\
\hline 9 & 17 & 1 & 0 & 11 & 2 & 0 & 12 & 3 & 2 & 12 & 3 & 0 & 7 & 2 & 0 \\
\hline 10 & 23 & 2 & 1 & 33 & 5 & 4 & 24 & 7 & 1 & 18 & 14 & 4 & 9 & 10 & 4 \\
\hline
\end{tabular}

I = granulomas classified as "not otherwise specified"; II = granulomas related to lymphatic vessels (adjacent, luminal, and distorting wall); III = granulomas related to blood vessels; $\mathrm{H}=$ haematoxylin; $\mathrm{E}=$ eosin.

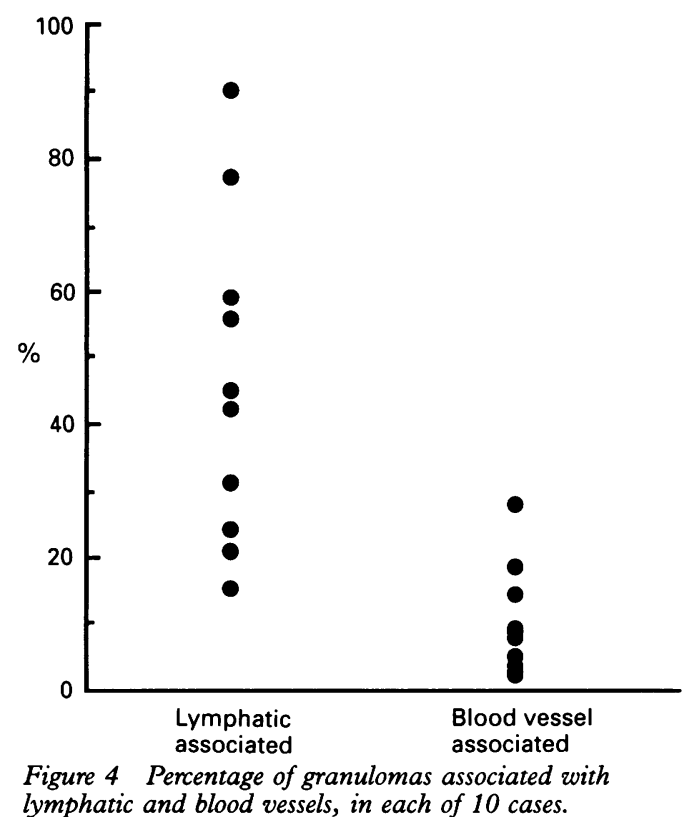

lymphatic and blood vessels, in each of 10 cases.

The percentages of the total number of granulomas which were related to lymphatic and blood vessels are shown in fig 4 . For lymphatic vessels, this figure ranged from 15.3 to $90.4 \%$, with a mean of $46 \cdot 1 \%$ and a median of $43.5 \%$. For blood vessels, the range was $2 \cdot 4$ to $25 \cdot 8 \%$ and the mean $10 \cdot 1 \%$ (median $8.4 \%$ ). There was no correlation between vascular and lymphatic involvement in individual cases.

Review of cases of ulcerative colitis revealed a total of three cellular aggregates in the lumina of lymphatic vessels in two cases, which were suggestive of granulomas. However, these aggregates showed a significant mixture of histiocytes and lymphocytes, rather than the peripheral rim of lymphocytes seen in Crohn's granulomas and in no case was disruption of the wall identified.

\section{Discussion}

Despite the characteristic pathological features of Crohn's disease, the aetiology of this condition remains obscure. Non-caseating granulomas are found in approximately $60 \%$ of cases of Crohn's disease ${ }^{7}$ and a small minority of cases show an arteritis. Granulomatous inflammation has been associated with this vasculitis in the gut ${ }^{8}$ and in lesions of Crohn's disease occurring elsewhere perivascular localisation of granulomas has been noted. ${ }^{910}$ Geller and Cohen ${ }^{3}$ claimed that $20 \%$ of cases of intestinal Crohn's disease showed arterial lesions, including granulomatous arteritis in $4 \%$. More recent studies have claimed that most of the granulomas form in the walls of blood vessels and that Crohn's disease is mediated by a vasculitis. ${ }^{11}$ Intravascular thrombosis is rare, however, and cases with a granulomatous vasculitis have not shown deposition of immunoglobulin or complement. ${ }^{12}$ Recognisable ischaemic changes were not seen in our specimens and the hypothesis that Crohn's disease is primarily a vascular disease has not found general acceptance. ${ }^{4-6}$

Lymphatic involvement has been recognised in Crohn's disease for some time, with dilated lymphatic vessels prominent in the submucosa. ${ }^{2}$ Lymphoid aggregates are frequently associated with these and granulomas adjacent to the dilated channels, pressing on their walls, have also been noted. ${ }^{13}$ The concept of Crohn's disease as a granulomatous lymphangitis was discussed by Hadfield in $1939^{14}$ and elaborated on by Warren and Somers, who referred to this as the "chief disease process". ${ }^{15}$ Among the pathogenic mechanisms suggested were sclerosis and obstruction of lymphatic vessels, with characteristic submucosal oedema and fibrosis. Also suggested was obstruction of arterial lumina by granulomatous inflammation located in adjacent lymphatic vessels, which seems less likely given arterial wall thickness. While granulomas are not identified in every case of Crohn's disease, they are likely to represent a significant feature in the pathogenesis of this disease, being "an adaptive mechanism for the removal or localisation of the causative agent of Crohn's disease". ${ }^{16}$ Despite the fact that granulomas are frequently associated with lymphoid aggregates, McGovern and Goulston felt that as the former were not invariably present and the latter were, and that granulomas were an epiphenomenon rather than an essential feature of the pathological process. ${ }^{8}$ By contrast, their importance, and possible primary significance, was emphasised by others. ${ }^{17}$

The distinction between small blood vessels and lymphatic vessels may be difficult on sections stained with haematoxylin and eosin ${ }^{18}$ and there may be considerable interobserver variation. ${ }^{19}$ Distinction between the two, paradoxically, may be easier in routinely processed tissue than in specimens treated by perfusion fixation, where the blood vessels remain dilated and their walls thin. ${ }^{1}$ Previous workers have used antibody panels similar to ours to differentiate between them. Factor VIII positivity has been reported as weak and focal in lymphatic vessels. ${ }^{1820}$ Ulex has been reported as showing 
a similar intensity of staining in blood and lymphatic vessels. ${ }^{1819}$ In our cases blood vessel staining was more intense, but variation in endothelial marker positivity is recognised. ${ }^{21}$ Collagen IV and laminin have variously been reported as being either weakly positive ${ }^{18}$ or negative ${ }^{20}$ in lymphatic capillaries, but both of these studies showed patchy weak positivity in lymph collecting vessels.

The combination of morphology and immunohistochemical profile of our cases enabled a satisfactory distinction to be made between lymphatic vessels, with definite endothelial and minimal basement membrane positivity, and blood vessels, with strong and distinct staining for both.

Although compact sarcoid-like granulomas were seen in many cases, most of the granulomas were small and loosely formed, with a pattern similar to that described in gastric Crohn's disease. ${ }^{5}$ The pattern of central aggregation of histiocytes, the presence of similar granulomas in tissue as well as lymphatic lumina and their absence in ulcerative colitis, where dilated lymphatics may also be seen, is proof that these are true granulomas, rather than intra-luminal cellular sediment. Furthermore, if those granulomas confined to the lumen are excluded from the data in the table, the mean number of granulomas associated with lymphatic vessels falls only slightly, from $46 \cdot 1$ to $40 \cdot 6 \%$.

The overall percentage of granulomas characterised as related to lymphatic vessels $(46 \cdot 1 \%)$, we feel, is most likely an underestimate. The difficulties involved in identifying lymphatic vessels are not entirely abolished by immunohistochemistry, and in this study, although the impression that a dilated and thin-walled vessel was lymphatic was strong, a granuloma was not classified as related to a lymphatic vessel unless supported by the staining pattern as described. Similarly, granulomatous inflammation with sclerosis would result in obliteration of lymphatic vessels and a further increase the false negative rate. We saw tiny foci of positivity for endothelial markers in some granulomas, but unless there was anidentifiable channel, the designation "not otherwise specified" was retained.

The results of this study suggest that granulomatous lymphangitis is a primary lesion of Crohn's disease and the consequence of the localisation of granulomatous inflammation is the submucosal oedema and fibrosis which gives rise to many of the characteristic clinical, radiological and histological features of the disease. The causative antigen of Crohn's disease may be taken up by macrophages which then enter the lymphatic vessels. Why localisation occurs here is unclear as yet. In this study $25 \%$ of lymphatic associated granulomas showed disruption of the vessel wall, suggesting that intralymphatic granulomatous inflammation is an early event. Whether this is because of uptake of antigen by lymphatic endothelium is unclear. Dilatation of these vessels may reflect the presence of granulomatous inflammation, or may be secondary to sclerosis. The findings suggest a secondary role for arteritis in the pathogenesis of Crohn's disease, although given the antigenic similarity of vascular and lymphatic endothelium, blood vessel involvement would be expected. This involvement could be on the basis of exposure of a shared antigen to immune assault, or extension of granulomatous inflammation-for example, from mural lymphatics of arterioles.

Crohn's disease is one of the major granulomatous diseases of obscure aetiology, the other being sarcoidosis. Of interest is the documented association between the granulomas of sarcoid and lymphatic vessels and vascular channels, ${ }^{22} 23$ and the suggestion that, as sarcoid granulomas follow the lymphatic vessels of the lung, the granulomatous inflammation of blood vessels occasionally observed in this condition is secondary to the spread of granulomas into vessels and other structures, rather than a primary vasculitic process. ${ }^{24}$ Identification of the reasons for granuloma localisation in lymphatic vessels will be of major importance in elucidating the pathogenesis of Crohn's disease.

1 Wakefield AJ, Sankey EA, Dhillon AP, Sawyerr AFM, More $\mathrm{L}$, Sim R, et al. Granulomatous vasculitis in Crohn's disease. Gastroenterology 1991;100:1271-87.

2 Petras RE. Non-neoplastic intestinal disease. In: Sternberg SS, ed. Diagnostic surgical pathology. Vol 2. New York: Raven Press, 1989:967-1014.

3 Geller SA, Cohen A. Arterial inflammatory-cell infiltration in Crohn's disease. Arch Pathol Lab Med 1983;107:473-5.

4 Roussel F. Vascular lesions in Crohn's disease [letter] Histopathology 1992;23:394-5.

5 Ectors NL, Dixon MF, Geboes KJ, Rutgeerts PJ, Desmet VJ, Vantrappen GR. Granulomatous gastritis: a morphological and diagnostic approach. Histopathology 1993;23:55-61.

6 Rhodes J, Thomas GAO. Smoking: good or bad for inflammatory bowel disease? [editorial]. Gastroenterology 1994;106:807-9.

7 Rosai J. Gastrointestinal tract/small bowel. In: Rosai J, ed. Ackerman's Surgical Pathology. Vol 1. St Louis: CV Mosby Co, 1989:522-56

8 McGovern VI, Goulston SJM. Crohn's Disease of the colon. Gut 1968;9:164-76.

9 Burgdorf W, Orkin M. Granulomatous perivasculitis in Crohn's Disease. Arch Dermatol 1981;117:674-5.

10 Tatnall FM, Dodd HJ, Sarkany I. Crohn's Disease with metastatic cutaneous involvement and granulomatous metastatic cutaneous involvement and
cheilitis. $₹ R$ Soc Med 1987;80:49-51.

11 Wakefield AJ, Sawyerr AM, Dhillon AP, Pittilo RM, Rowles PM, Lewis AAM, et al. Pathogenesis of Crohn's Disease; Multifocal gastrointestinal infarction. Lancet 1989;ii: 1057-62.

12 Slater DN, Waller PC, Reilly G. Cutaneous granulomatous vasculitis: presenting feature of Crohn's Disease. $\mathcal{F} R$ Soc Med 1985;78:589-90

13 Antonius J, Gump FE, Lattes R, Lepore M. Study of certain microscopic features in regional enteritis, and their possible prognostic significance Gastrenterology 1960;38: 889-905.

14 Hadfield G. The primary histological lesion of regional ileitis. Lancet 1939;ii:773-5.

15 Warren S, Sommers SC. Pathology of regional ileitis and ulcerative colitis. $¥ A M A$ 1954;154:189-93.

16 Chambers TJ, Morson BC. The granuloma in Crohn's Disease. Gut 1979;20:269-74.

17 Knutson H, Lunderquist A. Vascular changes in Crohn's Disease. Am $\mathcal{F}$ Radiol 1968;103:380-5.

18 Hultberg BM, Svanholm H. Immunohistochemical differentiation between lymphangiographically verified lymphatic vessels and blood vessels. Virchow Arch A Pathol Anat 1989;414:209-15.

19 Beckstead JH, Wood GS, Fletcher V. Evidence for the origin of Kaposi's sarcoma from lymphatic endothelium. $A m \mathcal{F}$ Pathol 1985;119:294-300.

20 Lee AKC, de Lillis RA, Silverman MC, Wolfe H. Lymphatic and blood vessel invasion in breast carcinoma: A useful prognostic indicator? Hum Pathol 1986;17:984-7.

21 Saigo PE, Rosen PP. The application of immunohistochemical stains to identify endothelial-lined channels in mammary carcinoma. Cancer 1987;59:51-4.

22 Takemura T, Matsui Y, Saki S, Mikami R. Pulmonary vascular involvement in sarcoidosis: a report of 40 autopsy vascular involvement in sarcoidosis: a

23 Colby TV, Lombard C, Yousem SA, Kitaichi M. Interstitial Diseases. In: Colby TV, et al, eds. Atlas of pulmonary surgical pathology. Philadelphia: WB Saunders Co, 1991: 227-306.

24 Churg A. Vasculitis in sarcoidosis. In: Churg A, Churg J, eds. Systemic vasculitides. New York: Igaku-Shoin, 1991: 299-304. 